\title{
Recombination landscapes in eight avian species
}

\author{
L. Malinovskaya ${ }^{1,2 *}, \mathrm{~N}$. Torgunakov ${ }^{2}$, A. Torgasheva ${ }^{1,2}$ \\ ${ }^{1}$ Institute of Cytology and Genetics SB RAS, Novosibirsk, Russia \\ ${ }^{2}$ Novosibirsk State University, Novosibirsk, Russia \\ *e-mail:l.malinovskaya@g.nsu.ru
}

Key words: avian chromosomes, recombination nodules, synaptonemal complex, MLH1

Motivation and Aim: Recombination is a key process of sexual reproduction. Shuffling the alleles, it increases genetic variation in populations and provides the raw material for natural selection. Recombination rate shows considerable interspecies variation, tightly linked with karyotypic variation in mammals. Relatively constant diploid chromosome number and inter-chromosomal conservation make birds an ideal model to study on evolution of recombination. However, up to date recombination landscapes have been described in a handful of bird species. Here we present a first description of the recombination characteristics of eight avian species from three orders.

Methods and Algorithms: Pachytene chromosome spreads were prepared from ovaries of nestling females and testes of adult males. To visualize recombination nodules and synaptonemal complex (SC) in pachytene cells we used immunolocalization of SYCP3, the main protein of the lateral element of the SC, MLH1, mismatch repair protein and centromere proteins.

Results: We found no significant variation of recombination rate across examined species. Recombination rate varied from 46.6 \pm 2.9 MLH1 foci per autosomal set in male sand martin $(2 n=80)$ to $55.1 \pm 5.2$ in female European pied flycatcher $(2 n=80)$. Interestingly, Eurasian hobby $(2 n=50)$ with the lower chromosome number had recombination rate similar to that in other species $(2 n \sim 80)$. We observed two different patterns of crossing over event distribution along the macrobivalents. The first one was characterized by recombination nodules more or less evenly distributed along the chromosome arms in male Eurasian Hobby, female barn swallow and European pied flycatcher. The second one was highly polarized with strong peaks at telomeric regions in male common swift, female great tit, female barn swallow, female and male sand and pale martins. In most cases, a chromosome morphology did not affect these patterns.

Conclusion: Our findings confirm the suggestion that birds have the higher level of recombination rate than mammals. Low interspecies variation of recombination rate may result from the relatively stable avian karyotype. However, we have found that reduction of chromosome number does not lead to decrease of recombination rate. This indicate that recombination rate might be constrained in birds by other factors. More data from different taxa are required to draw statistically supported conclusions about the evolution of recombination in birds.

Acknowledgements: The study was supported by the RFBR (17-29-08019, 18-04-00924) and the Federal Agency for Scientific Organizations via the Institute of Cytology and Genetics (Grant No. 0324-2018-0019). 